\title{
COMPUTATIONAL MODELING OF DIRECT PRINT MICROLITHOGRAPHY
}

\author{
A. A. DARHUBER*, S. M. MILLER*, S. M. TROIAN*, S. WAGNER ${ }^{+}$ \\ *Interfacial Science Laboratory, Dept. of Chemical Engineering, Princeton University \\ ${ }^{+}$Dept. of Electrical Engineering, Princeton University, Princeton, New Jersey 08544, USA
}

\begin{abstract}
Using a combination of experiment and simulations, we have studied the equilibrium shapes of liquid microstructures on flat but chemically heterogeneous substrates. The surface patterns, which define regions of different surface energy, induce deformations of the liquid-solid contact line, which in turn can either promote or impede capillary break-up and bulge formation. We study numerically the influence of the adhesion energies on the hydrophilic and hydrophobic surface areas, the pattern geometry and the deposited fluid volume on the liquid surface profiles.
\end{abstract}

\section{INTRODUCTION}

In the last decade many efforts have been made both to reduce the minimum feature size of electronic devices and to increase the throughput and reduce fabrication costs. Several groups have explored printing techniques such as gravure offset printing, screen printing, inkjet-printing and micro-contact printing for lithography or direct deposition of semiconductor- or polymerbased thin film transistors and light emitting diodes [1-11]. We are investigating wet printing techniques for the transfer of liquid inks from a chemically patterned surface onto an unpatterned target substrate. There are five major technological challenges involved in this process: (a) the fabrication of the printing plates, (b) the deposition and distribution of the ink on these stamps, (c) the control of the behavior of the ink patterns between ink deposition and printing, (d) the printing and (e) the stability of the printed ink patterns. In this article we focus on aspects of the behavior of liquid microstructures on chemically heterogeneous surfaces and the transfer process during printing.

\section{PRINTING PLATE FABRICATION}

The selective distribution of the ink on the imaging areas of the printing plate is achieved with the aid of a hydrophobic self-assembled monolayer (SAM) [12], which repels the ink from the non-imaging areas. There are several approaches to stamp fabrication. For example one can homogeneously coat a substrate like a silicon wafer with an SAM of octadecyltrichlorosilane (OTS) and subsequently remove the monolayer from the imaging areas by exposure to deep-UV light $(\lambda=193 \mathrm{~nm})$ through a chromium mask [13]. Alternatively, conventional photolithography can be used to pattern the SAM by first spin-coating photoresist on the SAM, exposing it to UV light through a chromium mask, developing the resist, removing the monolayer by oxygen plasma treatment and lastly removing the remaining photoresist. Since the mechanical resistance of SAMs to scratches is not very high and because scratches render the stamp surface hydrophilic in undesired locations, scratched stamps have to be reprocessed from the beginning or disposed. This problem can be partially overcome by depositing and patterning a $50 \mathrm{~nm}$ thick gold layer on the silicon surface by wet chemical etching and coating the gold with an SAM of hexadecanethiol (HDT). HDT forms a chemical bond with gold but does not adhere to the bare 
silicon regions. In case of surface scratches, which only damage the HDT layer, the sample only needs to be immersed in an HDT solution again, but no lithography step is required since the pattern is already defined in the gold layer. Kumar and Whitesides have developed another stamping technique called micro-contact printing, where a thiol-SAM is deposited on a flat gold surface by selective deposition with a polymer stamp [14].

\section{NUMERICAL PROCESS SIMULATIONS}

Numerical simulations of the equilibrium shapes of liquid microstructures have been performed with Surface Evolver [15], taking into account the surface tension $\gamma_{1 v}$ of the liquid and the contact angles on both the hydrophilic and hydrophobic parts of the surface. An example of a droplet on a hydrophilic channel, which spread over the channel boundaries, is presented in Fig. 1 along with a corresponding calculated droplet shape. A more extensive discussion of the simulation technique can be found in Ref. [16].
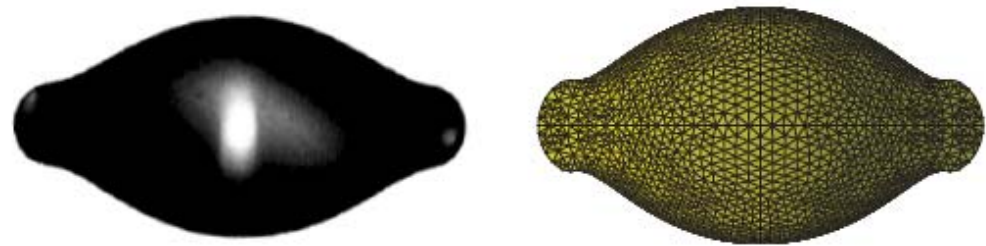

Figure 1: A droplet of glycerol (left, width $47 \mu \mathrm{m}$ ) on a hydrophilic channel defined with an OTS monolayer on a silicon wafer and a simulated shape (right).

Figure 2 shows top and side view profiles of ink lines at the junction of two hydrophilic channels, which meet at an angle of $60^{\circ}$. A corresponding volume filling factor $f$ may be defined as the ratio of the total ink volume and the product of the total hydrophilic surface area and the channel width. If the junction of the lines has an acute outer corner [Fig. 2(a),(b),(e),(f)], the liquid forms a bulge in the corner. Such an uneven height profile would be unfavorable for printing. If the outer corner is cut and the junction width is made narrower than the channel width [Fig. 2(c),(g)], the height profile is more or less even for a filling factor of $f=0.2$. However, if the volume is reduced by 50 percent [Fig. 2(d),(h)] the height of the liquid is reduced in the junction region. Upon further reduction of the volume the continuous liquid surface would pinch off at the corner. Thus, maintaining an even height profile requires both the proper design of the surface pattern and the control of the deposited liquid volume.

An explanation for the bulge formation is sketched in Fig. 3(a). Because of surface tension, the liquid prefers to reside in regions where it can minimize its surface curvature. Since the radius of a circle, which can be inscribed within the boundaries of the hydrophilic region, is

(a)

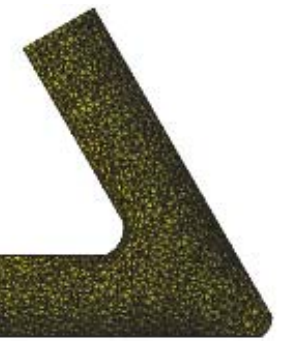

(e)

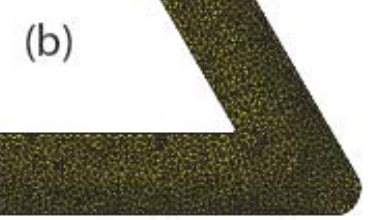

(f) (c)

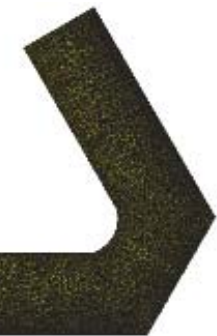

(g) (d)

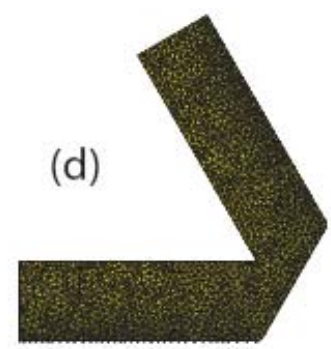

(h) 
Figure 2: Top and side-view profiles of ink lines at a junction of two hydrophilic channels, which meet at an angle of $60^{\circ}$. (a)-(d) are top views, (e)-(h) are side views along the plane of symmetry. Only one half of the surface profile is shown in the side views because of mirror symmetry. The volume filling factors are $f=0.2$ for (a), (c), (e) and (g) and $f=0.1$ for (b), (d), (f) and (h).
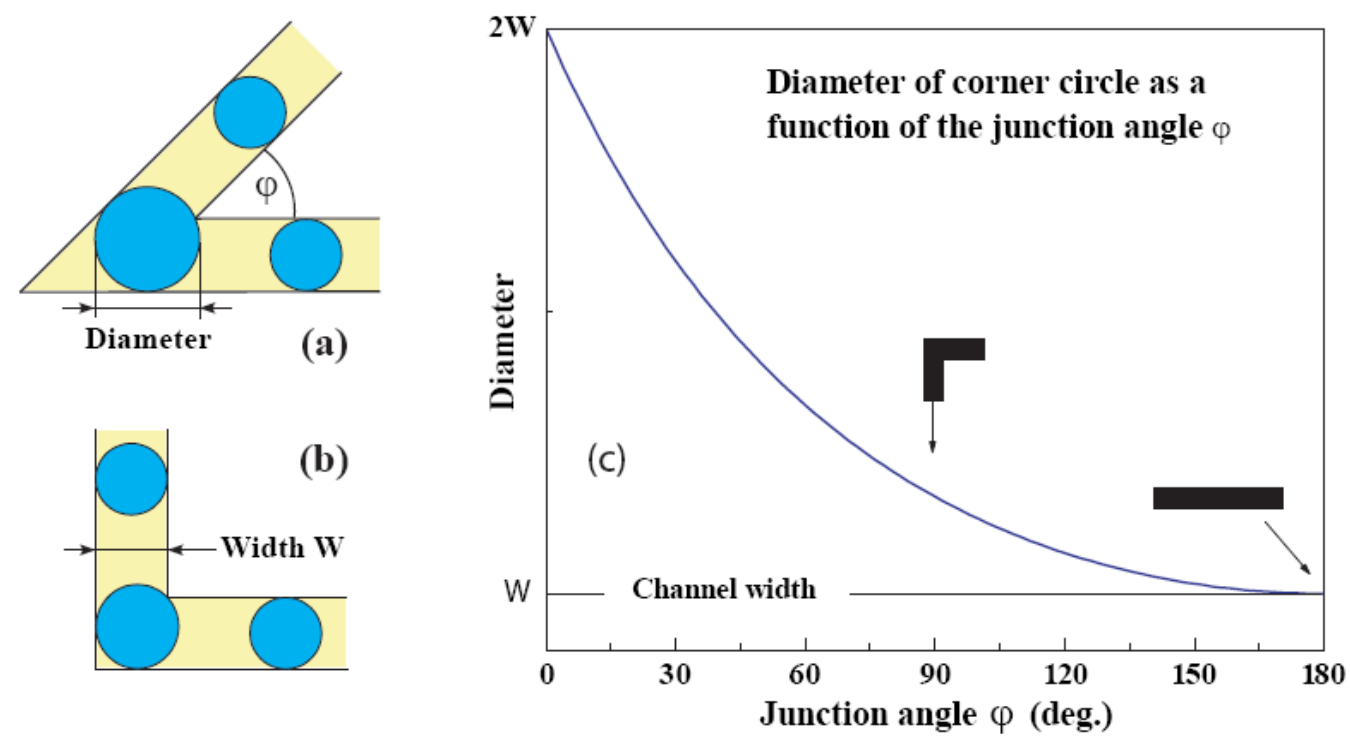

Figure 3: Qualitative explanation for the bulge formation in the junction region of two hydrophilic lines. (a,b) Sketches of the junction geometry for a junction angle $\varphi$ of (a) $60^{\circ}$ and (b) $90^{\circ}$. (c) The radius of the largest circle inscribed in the corner as a function of $\varphi$.

maximal in the junction region, the liquid will preferably accumulate there. This explanation is qualitative because the liquid can cross these boundaries and enter the interior hydrophobic region of the junction as shown in Figs. 2(a) and (c). Fig. 3(c) plots the radius of a circle inscribed in the corner as a function of the junction angle $\varphi$. As evident, the effect and thus the incentive for bulge formation becomes more pronounced for smaller values of $\varphi$.

In view of the trends shown in Figs. 2 and 3, the optimal channel geometry seems to be one in which the radius of an inscribed circle is constant. However, if the deposited liquid volume is very high, bulges will nonetheless form in the corner regions [17]. Thus, acute angles and small radii of curvature of the boundary lines between hydrophilic and hydrophobic regions should be avoided if pattern fidelity is to be maintained. When liquid is transferred to a nonporous substrate and the separation of the plates becomes small, the liquid will be squeezed between the plates beyond the boundaries of the hydrophilic regions. Therefore, the spacing of the printing plates must be controlled and maintained above a certain minimum value. A suitable solution is to place rigid spacer elements on the printing plate, which mechanically impede too close a contact between stamp and target surface. These spacers must be hydrophobic or they will attract ink during the deposition process. The spacer thickness must be tuned such that the contact line on the target substratematches the designed pattern on the stamp as closely as possible.

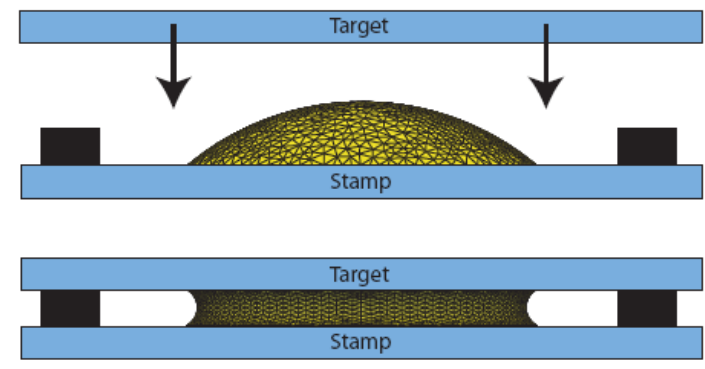

Figure 4: The use of spacer elements (black boxes) prevents the squeezing of the ink beyond the designed pattern boundaries. 
Since contact of the stamp and target plates leads to a redistribution of liquid, the required spacer thickness depends on the pattern geometry. Two limiting cases are straight, long lines, and circular pads. For the line geometry the redistribution of ink occurs only in the direction transverse to the channels, while for the circular pads, the ink spreads radially in-plane. Assuming identical ink profile heights for circles and lines, the plate separation required to maintain registry of designed and printed dimensions is smaller for circles than for lines.

Figure 5 shows the spacer thickness $s$ as a function of the apparent contact angle for infinite lines and circular pads. $s$ is given in units of the feature width, which corresponds to the diameter of the circular pads or the width of the lines. The apparent contact angle increases with the volume deposited on the surface pattern. As outlined above, the spacer thickness is smaller for circular pads than for long lines. Moreover, the spacer thickness $s$ depends on the contact angle on the target substrate $\theta_{\text {target}}$; the smaller $\theta_{\text {target, }}$ the larger is $s$. However, this dependence is weak for apparent contact angles below $40^{\circ}$ on the stamp. Therefore, the dimensions of the designed pattern may need to be corrected, if it contains both elongated and compact shapes.

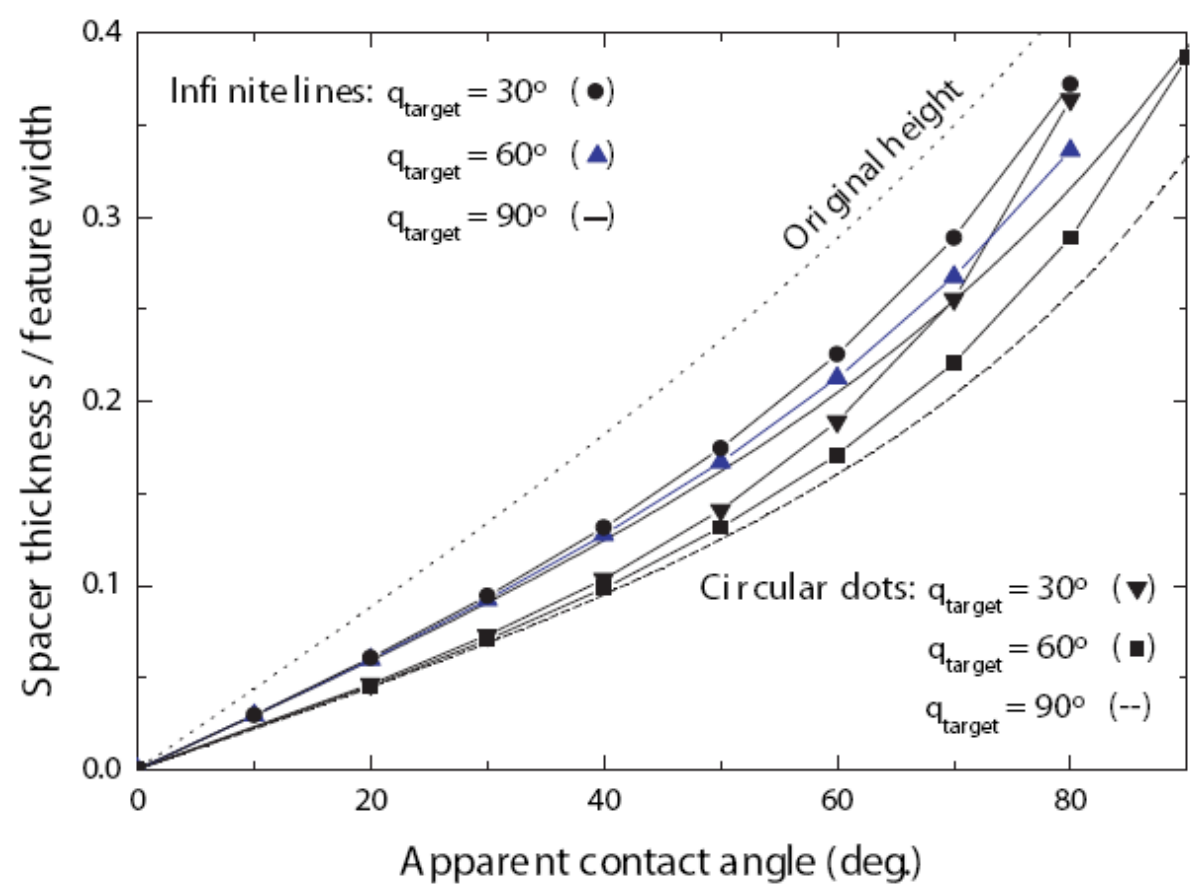

Figure 5: The required spacer thickness in units of the feature width as a function of the apparent contact angle for infinite lines and circular pads. The dotted line indicates the height of the lines and dots prior to contact with the target surface.

For the actual ink transfer, an important question is how the time-scale of the printing process (e.g. the time required to separate the printing plates) compares with the intrinsic time-scales of the liquid microstructures. These intrinsic time-scales are related both to the capillary relaxation time and the speed of the liquid-solid contact line motion. To obtain an estimate for the speed of capillary relaxation processes, we have investigated three models. The first was developed by S. E. Orchard for calculating the leveling time of periodic corrugations in paint films applied with a 
brush [18]. Assuming that the wavelength $\lambda$ of the corrugations is much larger than the average film thickness $h$, the final expression for the leveling time $\Delta \mathrm{t}$ is

$$
\Delta t=\frac{3}{16 \pi^{4}} \frac{\eta \lambda^{4}}{h^{3} \gamma_{l v}} \ln \left(\frac{a_{0}}{a}\right),
$$

where $\eta$ is the viscosity of the liquid, $a_{0}$ the initial amplitude of the corrugation and $a$ its amplitude after time $\Delta \mathrm{t}$. Inserting $\lambda=4 \mu \mathrm{m}, h=0.5 \mu \mathrm{m}, \gamma=0.07 \mathrm{~N} / \mathrm{m}, \eta=0.001 \mathrm{~Pa}$ s and $a_{0} / a=$ 10 results in $\Delta \mathrm{t}=1.3 \cdot 10^{-7} \mathrm{~s}$. The second model describes the spreading of a droplet on a smooth, flat and partially wetting surface [19]. It takes into account the friction processes associated with the contact line motion and gives similar results. The third model [20] describes the free oscillation of a spherical droplet, whose frequencies are given by

$$
\omega^{2}=\mathrm{n}(\mathrm{n}-1)(\mathrm{n}+2) \gamma /\left(\rho R^{3}\right) .
$$

Assuming a radius of $\mathrm{R}=1 \mu \mathrm{m}$ and a density of $\rho=1000 \mathrm{~kg} / \mathrm{m}^{3}$, gives a period of $\mathrm{T}=2.6 \cdot 10^{-7} \mathrm{~s}$.

Thus, for identical material parameters and similar feature sizes, all three models yield $\Delta \mathrm{t}$ of order $10^{-7} \mathrm{~s}$ for the capillary relaxation times. According to Eq. (1) $\Delta \mathrm{t}$ is proportional to $\eta$ and inversely proportional to $\gamma_{\mathrm{lv}}$. The viscosity of glycerol, which we use as a model ink because of its low vapor pressure, is about 2000 times higher and its the surface tension is $0.063 \mathrm{~N} / \mathrm{m}$. The maximum separation velocity of our laboratory printing press is $500 \mu \mathrm{m} / \mathrm{s}$, thus the time required to separate the plates by $2 \mu \mathrm{m}$ is longer than $4 \cdot 10^{-3} \mathrm{~s}$. Therefore, the printing process for micronsized glycerol lines and droplets is to be considered slow and determined by the chemical surface properties of the stamp and the target [21]. If the separation velocity, the ink viscosity or the feature size is significantly higher, the chemistry no longer determines the transfer process. We then enter the viscosity-controlled regime, in which the snap-off velocity of the ink meniscus is much higher than the liquid-solid contact line velocity on the plates and the transfer ratio of the ink is expected to become $50 \%$ irrespective of surface properties [22].

\section{SUMMARY}

We have presented numerical simulations of the equilibrium shapes of liquids on chemically patterned surfaces. From these model calculations we derived design rules for the plate fabrication, which include that connected regions of different width and acute junction angles of straight channels should be avoided. High curvatures of the boundary lines between hydrophilic and hydrophobic regions tend to become smoothened, particularly for higher deposited ink volumes. An estimate of the time-scales of the printing process allowed to identify and quantify the regimes where the ink transfer is governed by the chemical surface properties or by the dynamical material and process parameters.

\section{ACKNOWLEDGMENTS}

This work is supported by the Defense Advanced Research Projects Agency under the Molecular Level Printing Program. We are grateful to the Austrian Fonds zur Förderung der Wissenschaftlichen Forschung for a post-graduate fellowship (AAD) and the Eastman Kodak Corporation for a graduate fellowship (SMM). Surface Evolver was developed by Kenneth Brakke, Susquehanna University, Selinsgrove PA.

\section{REFERENCES}

[1] E. Kaneko, Electrochem. Soc. Proc. 96-23, 8 (1996).

[2] Y. Mikami et al., IEEE Transact. Electr. Dev. 41, 306 (1994). 
[3] H. Asada, H. Hayama, Y. Nagae, S. Okazaki, Y. Akimoto, T. Saito, Conference Record of the 1991 Int. Display Research Conference, p. 227 (1991)

[4] F. Garnier, R. Hajlaoui, A. Yassar, P. Srivastava, Science 265, 1684 (1994).

[5] B. A. Ridley, B. Nivi, J. M. Jacobson, Science 286, 746 (1999).

[6] T.-X. Liang, W. Z. Sun, L.-D. Wang, Y. H. Wang, H.-D. Li, IEEE Transact. Components, Packaging and Manufacturing Technology B19, 423 (1996).

[7] Z. Bao, Y. Feng, A. Dodabalapur, V. R. Raju, A. Lovinger, Chem. Mater. 9, 1299 (1999).

[8] J. A. Rogers, Z. Bao, A. Makhija, P. Braun, Adv. Mater. 11, 741 (1999).

[9] Z. Bao, Adv. Mater. 12, 227 (2000).

[10] T. R. Hebner, C. C. Wu, D. Marcy, M. H. Lu, J. C. Sturm, Appl. Phys. Lett. 72, 519 (1998).

[11] J. Bharathan, Y. Yang, Appl. Phys. Lett. 72, 2660 (1998).

[12] J. D. Swalen, D. L. Allara, J. D. Andrade, E. A. Chandross, S. Garoff, J. Israelachvili, T. J. McCarthy, R. Murray, R. F. Pease, J. F. Rabolt, K. J. Wynne, H. Yu, Langmuir 3, 932 (1987).

[13] C. S. Dulcey, J. H. Georger, V. Krauthamer, D. A. Stenger, T. L. Fare and J. M. Calvert, Science 252, 551 (1991).

[14] A. Kumar, G. M. Whitesides, Appl. Phys. Lett. 63, 2002 (1993).

[15] K. Brakke, Experimental Mathematics 1, 141 (1992).

[16] A. A. Darhuber, S. M. Troian, S. M. Miller, S. Wagner, J. Appl. Phys. (2000), in press.

[17] H. Gau, S. Herminghaus, P. Lenz and R. Lipowsky, Science 283, 46 (1999).

[18] S. E. Orchard, Appl. Sci. Res. A11, 451 (1962).

[19] M. J. de Ruijter, M. Charlot, M. Voue, J. De Coninck, Langmuir 16, 2363 (2000); M. J. de Ruijter, J. De Coninck, G. Oshanin, Langmuir 15, 2209 (1999).

[20] H. Lamb, Hydrodynamics, Dover Publications (New York, 1945).

[21] A. A. Darhuber, S. M. Troian, S. M. Miller, S. Wagner, Proc. of the $3^{\text {rd }}$ Int. Conf. on Modeling and Simulation of Microsystems (San Diego, March 2000), Computational Publications (Cambridge, 2000).

[22] E. D. Yakhnin, A. V. Chadov, Kolloidnyi Zhurnal 45, 1183 (1983). 